\title{
Synergistic Antibacterial Activity Between 1,4-Naphthoquinone and $\beta$-Lactam Antibiotics Against Methicillin-Resistant Staphylococcus aureus
}

\author{
Jeremy Kean Yi Yap, Sharon Yoke Ying Tan, ${ }^{2}$ Shi Qi Tang, ${ }^{2}$ Vui Kien Thien, ${ }^{2}$ and Elaine Wan Ling Chan ${ }^{3}$
}

\begin{abstract}
Aims: Currently, limited antibiotics are available to treat methicillin-resistant Staphylococcus aureus (MRSA) infections. One approach is the use of adjuvants in antibiotic therapy. 1,4-Naphthoquinones are naturally occurring alkaloids shown to have antibacterial properties. The objective of this study is to investigate the synergy between 1,4-naphthoquinone and selected $\beta$-lactam antibiotics and to evaluate the potential use of 1,4naphthoquinone as an adjuvant in antibiotic treatment against MRSA infections.

Methods: The antibacterial activity of 1,4-naphthoquinone and plumbagin was tested against nine pathogenic bacterial strains using the microdilution broth method. The interactions between 1,4-naphthoquinone and three antibiotics (cefuroxime, cefotaxime, and imipenem) were estimated by calculating the fractional inhibitory concentration of the combination.

Results: The compounds 1,4-naphthoquinone and plumbagin exhibited a broad range of bacteriostatic and bactericidal effects against both Gram-positive and Gram-negative bacteria. The interaction between 1,4naphthoquinone and imipenem, cefuroxime, and cefotaxime was synergistic against methicillin-sensitive Staphylococcus aureus and MRSA clinical strains. Against ATCC-cultured MRSA, a synergistic effect was observed between 1,4-naphthoquinone and cefotaxime. However, combination with imipenem only produced an additive effect, and an antagonistic action was observed between 1,4-naphthoquinone and cefuroxime.

Conclusions: Although individually less potent than common antibiotics, 1,4-naphthoquinone acts synergistically with imipenem, cefuroxime, and cefotaxime against MRSA clinical strains and could potentially be used in adjuvant-antibiotic therapy against multidrug resistant bacteria.
\end{abstract}

Keywords: 1,4-naphthoquinone, adjuvant, Staphylococcus aureus, cefuroxime, cefotaxime, imipenem

\section{Introduction}

T HE DISCOVERY OF ANTIBIOTICS has transformed the clinical armamentarium, as well as the pharmaceutical industry. However, over time, bacteria develop resistance toward these antibiotics, making them less effective medical therapies for bacterial infections. ${ }^{1}$ It is apparent that antibiotic resistance is driven by the overuse and inappropriate use of antibiotics. With the discovery of new antibiotics, antibiotic therapy can once again be made effective to treat infections caused by multidrug-resistant bacteria. However, due to poor control over the evolutionary properties of bacteria, antibiotic resistance emerges more rapidly than new antibiotics are discovered. ${ }^{2}$ Overwhelming regulatory, economic, and scientific challenges brought about by antibiotic resistance further complicate the development of novel antibiotics. As such, alternative strategies must be sought to halt the rapid emergence of antibiotic resistance.

The use of adjuvants to improve the efficacy of antibiotics against drug-resistant bacteria is an attractive strategy to minimize the requirement for new antibiotics. ${ }^{3}$ The antibiotic-adjuvant combination approach confers several advantages over the development of new antibiotics. For example, the combination of an antibiotic with an adjuvant compound could reduce the opportunity for bacteria to develop resistance. The mechanism of action of such adjuvantantibiotic drugs primarily involves disabling the defence mechanism of bacteria toward the antibiotics, thus restoring

\footnotetext{
${ }^{1}$ School of Postgraduate Studies, ${ }^{2}$ School of Health Sciences, and ${ }^{3}$ Institute for Research, Development and Innovation, International
} Medical University, Kuala Lumpur, Malaysia. 
bacterial susceptibility. An example of a popular adjuvantantibiotic drug is the combination of amoxicillin and a $\beta$-lactamase inhibitor known as clavulanic acid. Clavulanic acid is marketed under the trade name Augmentin and used to treat a variety of common infections, including urinary tract infections, upper and lower respiratory tract infections, otorhinolaryngological infections, and skin and soft-tissue infections. ${ }^{4}$ Commercialization of adjuvant-antibiotic drugs such as Augmentin further inspired the production of other adjuvant-antibiotic therapies to treat a wide range of antibiotic-resistant bacteria. ${ }^{5}$

Naphthoquinone-producing plants have been exploited for centuries for their numerous pharmacological applications. ${ }^{6}$ Quinoid compounds are in use clinically, including chemotherapeutic anthraquinones such as doxo- and daunorubicin and the mitomycins. ${ }^{7}$ Several compounds with the backbone chemical structure of 1,4-naphthoquinone have been shown to be nontoxic to humans as these compounds do not alter the coagulation parameters in human plasma. ${ }^{8}$ The organic compound 1,4-naphthoquinone could be a promising substance in adjuvant development as 1,4naphthoquinone and its derivatives, such as plumbagin, have been shown to exhibit antibacterial effects especially against Staphylococcus aureus. ${ }^{9-11}$ Besides antibacterial activity, 1,4-naphthoquinone and its derivatives also exhibit antifungal activity against Candida krusei, Candida parapsilosis, and Cryptococcus neoformans, ${ }^{6,12-14}$ antiinflammatory activities, ${ }^{6,15}$ antiparasitic properties, ${ }^{6,16}$ and anticancer properties. ${ }^{17}$ Since inflammation is commonly associated with bacterial infections, the anti-inflammatory properties of this compound could provide an additional advantage. Given its putative usefulness, the aim of this study is to investigate the antibacterial effects of 1,4naphthoquinone against several pathogenic Gram-positive and Gram-negative bacteria, following which the potential of 1,4-naphthoquinone to be used as an antibiotic adjuvant was assessed by examining its capability to reverse the resistance of methicillin-resistant Staphylococcus aureus (MRSA) to selected $\beta$-lactam antibiotics, namely cefuroxime, cefotaxime, and imipenem.

\section{Materials and Methods}

\section{Chemical compounds and standard antibiotics}

The chemical compounds 1,4-naphthoquinone and plumbagin were obtained in powder form from Merck KGaA (Germany) and Sigma-Aldrich, respectively. The antibiotics imipenem, cefuroxime, and cefotaxime, in powder form, were obtained from Merck Sharp and Dohme Corp., CCM Duopharma Biotech Berhad (Malaysia), and Fisher Scientific, respectively.

\section{Bacterial strains}

The bacterial strains used included: (1) Gram-positive bacteria: Micrococcus luteus (ATCC 4698), Bacillus cereus (ATCC 14579), methicillin-sensitive Staphylococcus aureus (MSSA) (ATCC 25923), MRSA (ATCC 33591), two hospital isolates of MRSA; and (2) Gram-negative bacteria: Escherichia coli (ATCC 25922), Klebsiella pneumoniae (ATCC 10031; ATCC 2146; ATCC 1705), Pseudomonas aeruginosa (ATCC 10145), and Salmonella choleraesuis
(ATCC 10708). All strains of bacteria used were subcultured from our laboratory stock culture collection, including clinical isolates of MRSA.

\section{Antibacterial activity and susceptibility tests}

The antibacterial activities of both 1,4-naphthoquinone and plumbagin were determined using the microdilution method as described by the Clinical and Laboratory Standards Institute. ${ }^{18}$ The minimum inhibitory concentration (MIC), which determines the lowest concentration of the compound that inhibits bacterial growth completely, was performed in four replicates. A two-fold serial dilution was performed using cation-adjusted Mueller-Hinton broth (CA$\mathrm{MHB}$ ) with a concentration ranging from 0.0005 to $1.0 \mathrm{mg} / \mathrm{mL}$. Bacteriostatic effects indicated by the MIC were obtained after incubation at $37^{\circ} \mathrm{C}$ for $24 \mathrm{hr}$. Cleared wells with no visible growth were subcultured $(0.1 \mathrm{~mL})$ onto a nutrient agar plate and incubated overnight at $37^{\circ} \mathrm{C}$ to determine the minimum bactericidal concentration (MBC). The MBC is the lowest concentration at which no visible growth is observed on agar plates.

\section{Fractional inhibitory concentration index}

A checkerboard method was used to examine the additive, synergistic, or antagonistic effects of cefuroxime, cefotaxime, and imipenem when used in combination with 1,4-naphthoquinone. The effects were determined by calculating the fractional inhibitory concentration index (FICI) for each replicate using the following formula:

$$
\begin{gathered}
\text { FICI }=(\text { concentration of } \mathrm{A} \text { in combination } / \text { MIC of A alone }) \\
+(\text { concentration of } \mathrm{B} \text { in combination } / \\
\text { MIC of B alone })
\end{gathered}
$$

where A: 1,4-naphthoquinone, and B: antibiotic (imipenem, cefuroxime, or cefotaxime).

The combined effect of two compounds was considered to be synergistic when the FICI was $\leq 0.5$, additive when the FICI was $>0.5$ and $<2$, and antagonistic when the FICI was $\geq 2$. $^{19}$

The checkerboard method was performed in CA-MHB where dilutions of the 1,4-naphthoquinone and antibiotics were made on the basis of the MIC of these two compounds. The MIC of the combination of 1,4-naphthoquinone and antibiotics was obtained following an overnight incubation at $37^{\circ} \mathrm{C}$. Each test was performed in triplicate, and the results were represented by an isobologram with 1,4naphthoquinone on the $x$-axis and the respective antibiotics on the $y$-axis. A synergistic effect $(\mathrm{FICI} \leq 0.5)$ between the two compounds is indicated as a concave curve, additive $(0.5<$ FICI <2) interactions are represented by a straight line, and a convex curve indicates antagonism (FICI $\geq 2$ ).

\section{Results}

\section{Antibacterial activity of 1,4-naphthoquinone and plumbagin}

The chemical structures of (i) Plumbagin and (ii) 1,4naphthoquinone are shown in Fig. 1. Plumbagin (5- 
<smiles>CC1=CC(=O)c2c(O)cccc2C1=O</smiles>

i) Plumbagin

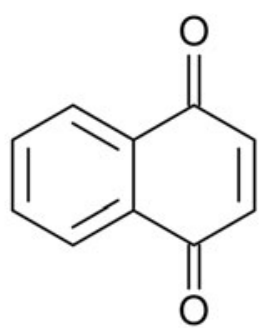

ii) 1,4-naphthoquinone
FIG. 1. The chemical structure of (i) Plumbagin and (ii) 1,4-naphthoquinone.

Hydroxy-2-methyl-1,4-naphthoquinone) is a derivative of 1,4-naphthoquinone that contains additional hydroxyl and methyl groups. From Table 1, both 1,4-naphthoquinone and plumbagin exhibited bacteriostatic and bactericidal effects against Gram-positive and Gram-negative bacterial strains except for $P$. aeruginosa. There was no bactericidal effect observed at the highest tested concentration of $1.0 \mathrm{mg} / \mathrm{mL}$. The MIC values of 1,4-naphthoquinone and plumbagin range from 0.0078 to $0.125 \mathrm{mg} / \mathrm{mL}$ and 0.0078 to $0.25 \mathrm{mg} / \mathrm{mL}$, respectively. Plumbagin has shown potent antibacterial activity (MIC $\leq 0.0313 \mathrm{mg} / \mathrm{mL}$ ) against $S$. aureus strains compared with 1,4-naphthoquinone ( $\mathrm{MIC}=0.125$ $\mathrm{mg} / \mathrm{mL}$ ). M. luteus was the most susceptible bacterium toward the two compounds $(\mathrm{MIC}=0.0078 \mathrm{mg} / \mathrm{mL}, \mathrm{MBC}=$ $0.0313 \mathrm{mg} / \mathrm{mL}$ ). The Gram-positive bacterium Enterococcus faecalis $(\mathrm{MIC}=0.0313 \mathrm{mg} / \mathrm{mL})$ and the Gram-negative bacteria $P$. aeruginosa, K. pneumoniae $1031, K$. pneumoniae 1705, E. coli, and Salmonella choleraesuis ( $\mathrm{MIC}=0.0625$ $\mathrm{mg} / \mathrm{mL}$ ) were more susceptible to 1,4-naphthoquinone than plumbagin, with MIC values ranging from 0.125 to $0.25 \mathrm{mg} / \mathrm{mL}$.

\section{Combinational effects of 1,4-naphthoquinone with the antibiotics imipenem, cefuroxime, and cefotaxime}

The combination effects of 1,4-naphthoquinone and imipenem, cefuroxime, or cefotaxime were examined using the checkerboard method. The calculated FICI determined the combination effects to be additive, synergistic, or antagonistic. From Table 2, synergistic interactions (FICI $\leq 0.5$ ) against MSSA ATCC 25923 and both clinical strains of MRSA were observed when 1,4-naphthoquinone was used in combination with imipenem, cefuroxime, and cefotaxime, as represented by a concave isobologram curve (Fig. 2). However, against ATCC cultured MRSA, a synergistic effect was observed between 1,4-naphthoquinone and cefotaxime $(\mathrm{FICI}=0.5)$, whereas the combination with imipenem only produced an additive effect $(\mathrm{FICI}=1.063)$, represented by a straight-line isobologram (Table 2; Fig. 2). In contrast, an antagonistic effect was observed between 1,4-naphthoquinone and cefuroxime ( $\mathrm{FICI}=2.25$ ) against ATCC cultured MRSA, with a corresponding convex isobologram curve (Table 2; Fig. 2).

\section{Discussion}

In this study, the antibacterial activity of 1,4-naphthoquinone with that of plumbagin (derivatives of 1,4-naphthoquinone) and the potential of 1,4-naphthoquinone as an antibiotic adjuvant against MRSA were examined. 1,4Naphthoquinone and plumbagin exhibited broad-spectrum inhibitory activity against both Gram-positive and Gramnegative bacteria at concentrations $<1 \mathrm{mg} / \mathrm{mL}$ (Table 1). This is in accordance with a previous study; 1,4-naphthoquinone and its derivatives displayed antibacterial activity against bacteria such as $S$. aureus and $P$. aeruginosa. ${ }^{10,20}$ Generally, a higher concentration of 1,4-naphthoquinone or plumbagin was required to kill Gram-negative bacteria than

Table 1. Minimum Inhibitory Concentration and Minimum Bactericidal Concentration of 1,4-Naphthoquinone and Plumbagin

\begin{tabular}{|c|c|c|c|c|}
\hline \multirow[b]{2}{*}{ Bacteria } & \multicolumn{2}{|c|}{ 1,4-Naphthoquinone } & \multicolumn{2}{|c|}{ Plumbagin } \\
\hline & $M I C(m g / m L)$ & $M B C(m g / m L)$ & $M I C(m g / m L)$ & $M B C(m g / m L)$ \\
\hline \multicolumn{5}{|l|}{ Gram-positive } \\
\hline Bacillus cereus ATCC 14579 & 0.0625 & 1.0 & 0.0625 & 0.125 \\
\hline Micrococcus luteus ATCC 4698 & 0.0078 & 0.0313 & 0.0078 & 0.0313 \\
\hline MSSA ATCC 25923 & 0.125 & 0.125 & 0.0313 & 0.0625 \\
\hline MRSA ATCC 33591 & 0.125 & 0.125 & 0.0313 & 0.5 \\
\hline MRSA clinical strain 1 & 0.125 & 0.125 & 0.0078 & 0.0625 \\
\hline MRSA clinical strain 2 & 0.125 & 0.125 & 0.0078 & 0.0313 \\
\hline Enterococcus faecalis ATCC 29212 & 0.0313 & 1.0 & 0.125 & 0.5 \\
\hline \multicolumn{5}{|l|}{ Gram-negative } \\
\hline Pseudomonas aeruginosa ATCC 10145 & 0.0625 & $>1.0$ & 0.125 & $>1.0$ \\
\hline Klebsiella pneumoniae ATCC 10031 & 0.0625 & 0.5 & 0.0625 & 1.0 \\
\hline Klebsiella pneumoniae ATCC 1031 & 0.0625 & 1.0 & 0.25 & 1.0 \\
\hline Klebsiella pneumoniae ATCC 2146 & 0.0625 & 0.5 & 0.0625 & 0.5 \\
\hline Klebsiella pneumoniae ATCC 1705 & 0.0625 & 0.5 & 0.125 & 1.0 \\
\hline Escherichia coli ATCC 25922 & 0.0625 & 0.125 & 0.25 & 1.0 \\
\hline Salmonella choleraesuis ATCC 10708 & 0.0625 & 0.125 & 0.125 & 0.25 \\
\hline
\end{tabular}

MBC, minimum bactericidal concentration; MIC, minimum inhibitory concentration; MRSA, methicillin-resistant Staphylococcus aureus; MSSA, methicillin-sensitive Staphylococcus aureus. 
Table 2. The Synergistic Effect of 1,4-Naphthoquinone and the Selected $\beta$-Lactam ANTIBIOTICS INDICATED by Fractional INHIBITORY CONCENTRATION INDICES

\begin{tabular}{|c|c|c|c|c|c|c|c|c|}
\hline \multirow[b]{2}{*}{ Bacteria } & \multicolumn{2}{|c|}{ Compound } & \multicolumn{2}{|c|}{ MIC } & \multicolumn{2}{|c|}{$F I C$} & \multirow[b]{2}{*}{ FICI } & \multirow[b]{2}{*}{ Interpretation } \\
\hline & $A$ & $B$ & $\begin{array}{c}M I C A \\
(\mu g / m L)\end{array}$ & $\begin{array}{c}M I C B \\
(\mu g / m L)\end{array}$ & FIC A & FIC B & & \\
\hline \multirow{3}{*}{$\begin{array}{l}\text { MSSA ATCC } \\
25923\end{array}$} & 1,4-Naphthoquinone & Imipenem & 125 & 64 & 0.625 & 0.625 & 0.125 & Synergistic \\
\hline & 1,4-Naphthoquinone & Cefuroxime & 125 & 64 & 0.0625 & 0.25 & 0.3125 & Synergistic \\
\hline & 1,4-Naphthoquinone & Cefotaxime & 125 & 2.0 & 0.03125 & 0.5 & 0.5 & Synergistic \\
\hline \multirow{3}{*}{$\begin{array}{l}\text { MRSA ATCC } \\
33591\end{array}$} & 1,4-Naphthoquinone & Imipenem & 125 & 0.5 & 0.625 & 1.0 & 1.063 & Additive \\
\hline & 1,4-Naphthoquinone & Cefuroxime & 125 & 1.0 & 0.25 & 2.0 & 2.25 & Antagonistic \\
\hline & 1,4-Naphthoquinone & Cefotaxime & 125 & 16 & 0.03125 & 0.5 & 0.5 & Synergistic \\
\hline \multirow{3}{*}{$\begin{array}{l}\text { MRSA clinical } \\
\text { strain } 1\end{array}$} & 1,4-Naphthoquinone & Imipenem & 125 & 128 & 0.03125 & 0.5 & 0.5 & Synergistic \\
\hline & 1,4-Naphthoquinone & Cefuroxime & 125 & 4096 & 0.0625 & 0.00195 & 0.06445 & Synergistic \\
\hline & 1,4-Naphthoquinone & Cefotaxime & 125 & 4096 & 0.03125 & 0.03125 & 0.0625 & Synergistic \\
\hline \multirow{3}{*}{$\begin{array}{l}\text { MRSA clinical } \\
\text { strain } 2\end{array}$} & 1,4-Naphthoquinone & Imipenem & 125 & 256 & 0.03125 & 0.5 & 0.5 & Synergistic \\
\hline & 1,4-Naphthoquinone & Cefuroxime & 125 & 4096 & 0.0625 & 0.00049 & 0.06299 & Synergistic \\
\hline & 1,4-Naphthoquinone & Cefotaxime & 125 & 4096 & 0.0625 & 0.00781 & 0.07031 & Synergistic \\
\hline
\end{tabular}

A: 1,4-naphthoquinone; B: antibiotics.

FICI $=$ total of combination cefuroxime and 1,4-naphthoquinone.

Interpretation: FICI $\leq 0.5$, synergistic; FICI $0.5<x<2$, additive; and FICI $\geq 2$ antagonistic. $^{19}$

FIC, fractional inhibitory concentration; FICI, fractional inhibitory concentration index; MIC, minimum inhibitory concentration.

Gram-positive bacteria. This is as expected because Gramnegative bacteria possess an outer lipopolysaccharide membrane in addition to a peptidoglycan wall, which encumbers compound diffusion into the cells. The presence of this lipopolysaccharide barrier protects the bacteria from antibacterial agents that can damage its peptidoglycan wall. Thus, Gram-negative bacteria naturally have better resistance against antibiotics than their Gram-positive counterparts.

In terms of bactericidal effects, both 1,4-naphthoquinone and plumbagin exhibited effects against all tested bacteria except for $P$. aeruginosa at a slightly higher concentration of up to $1 \mathrm{mg} / \mathrm{mL}$. Interestingly, a higher concentration of 1,4naphthoquinone is not necessary to kill MSSA and MRSA strains, as indicated by the same MIC and MBC value of $0.125 \mathrm{mg} / \mathrm{mL}$. The MBC of 1,4-naphthoquinone against MSSA and MRSA was among the lowest $(0.125 \mathrm{mg} / \mathrm{mL})$, similar to the MBC against $E$. coli and $S$. choleraesuis, and only outperformed by M. luteus $(0.0313 \mathrm{mg} / \mathrm{mL})$. A previous study showed that the mode of action for the antibacterial activity of 1,4-naphthoquinone and its derivatives involved enhanced reactive oxygen species (ROS) generation, which is followed by apoptotic cell death. The generation of ROS leads to the restriction of cellular enzymes, which are responsible for apoptotic cell death. ${ }^{11}$ Furthermore, a separate study showed that the carbonyl groups at $\mathrm{C} 1$ and $\mathrm{C} 4$ of naphthoquinones enhanced its antimicrobial activity. The two rings of the naphthoquinone core are essential for the antimicrobial activity of these compounds. In addition, the halogen substituents at the $\mathrm{C} 2$ and/or $\mathrm{C} 3$ positions are the functional groups that influence the biological activity of naphthoquinones (Fig. 1). However, the presence of additional rings in the structure or the lack of either ring will result in the inactivation of the compound. ${ }^{12}$ These studies suggest that 1,4-naphthoquinone and its derivatives could have great potential in the development of antibacterial drugs.

MRSA is known to be notoriously resistant to virtually every type of nonexperimental $\beta$-lactam antibiotic avail- able. ${ }^{21}$ The mechanism of resistance development in MRSA involves the acquisition of extrachromosomal genetic elements containing resistance genes, particularly the mecA gene encoding a low-affinity penicillin-binding protein $2 \mathrm{~A}$, which can be disseminated between bacteria through horizontal gene transfer. ${ }^{21-23}$ The antibiotics used in this study (imipenem, cefuroxime, and cefotaxime) were ineffective against MRSA infections, as evidenced by the exceptionally high MIC of these antibiotics against clinical isolates of MRSA (Table 2). In the present study, 1,4-naphthoquinone was generally shown to function synergistically in combination with most $\beta$-lactam antibiotics used against MSSA and MRSA. The synergism between 1,4-naphthoquinone and antibiotics against MSSA suggests that, with the use of 1,4-naphthoquinone as an adjuvant, a lower dose of antibiotics is required to treat nondrug-resistant $S$. aureus infections. This could be helpful to mitigate resistance development by limiting the amount of antibiotics used. This synergistic effect observed between an adjuvant and an antibiotic was also previously shown in studies whereby nonsteroidal anti-inflammatory drugs in combination with $\beta$-lactam antibiotics were able to increase their antibacterial activities against MRSA. ${ }^{24,25}$ Furthermore, another study showed that the antibacterial activity of Sophoraflavanone B against MRSA can be increased effectively through its combination with antibiotics such as $\beta$-lactams, aminoglycosides, and quinolones. These examples further indicate that besides the commercially successful use of the Augmentin combination (amoxicillin/clavulanate potassium), other compounds could potentially be used as an adjuvant to treat MRSA infections.

However, in ATCC-cultured MRSA, a limited response toward the combination treatments was observed. While the combination of 1,4-naphthoquinone and cefotaxime produced a synergistic effect, combining 1,4-naphthoquinone with imipenem only generated an additive effect, suggesting that the two compounds do not chemically augment each other's antibacterial capacity. Combination with cefuroxime 


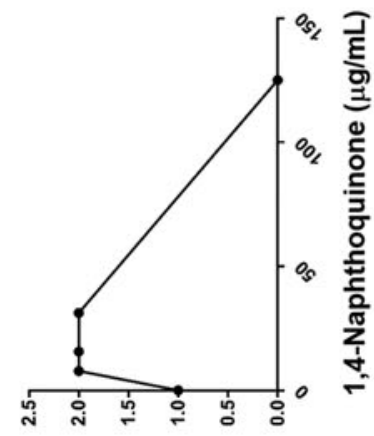

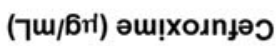

$\boldsymbol{m}$

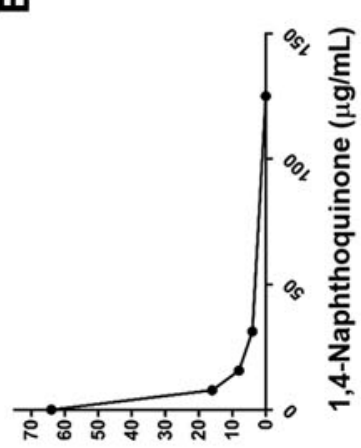

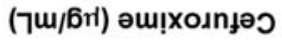

$\varangle$

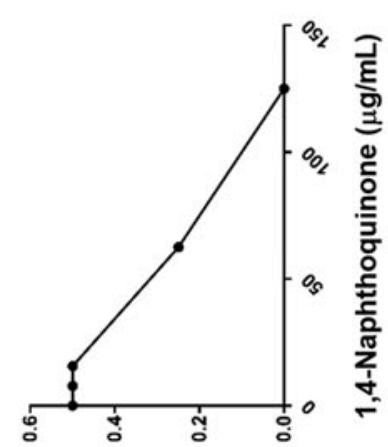

(ךu/6̂r) məuəd!̣u।

m

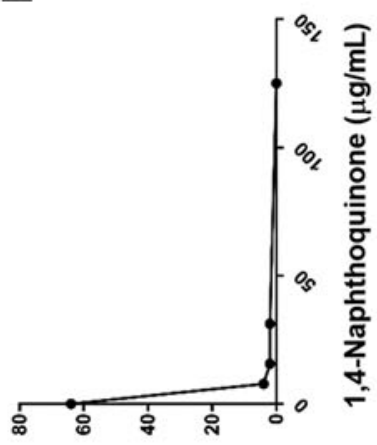

(ךu/6̂t') məuəd!̣u|

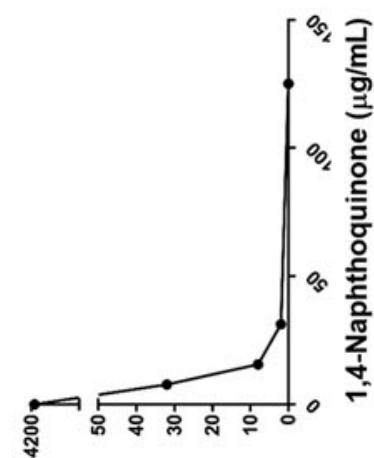

(ךu/6ri) әแ!̣xoגกృəว

อ
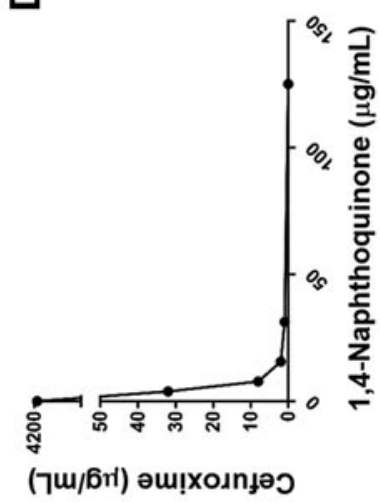

0

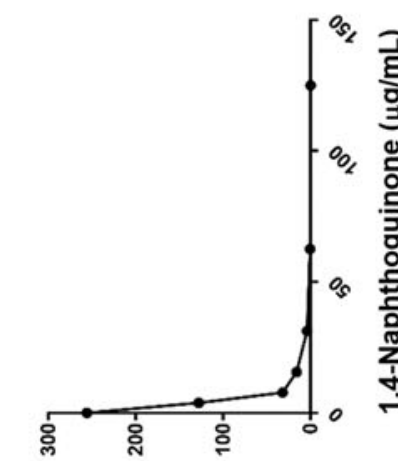

(ךu/б̂n') məuəd!̣u|

口

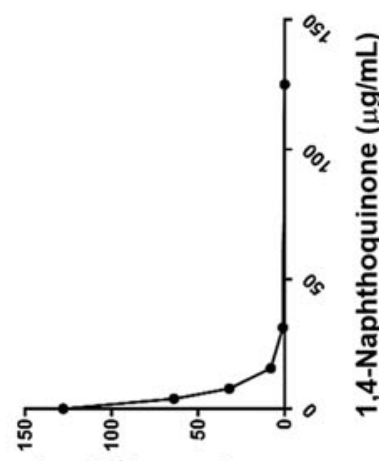

(ךu/6ril) məuəd!̣u।

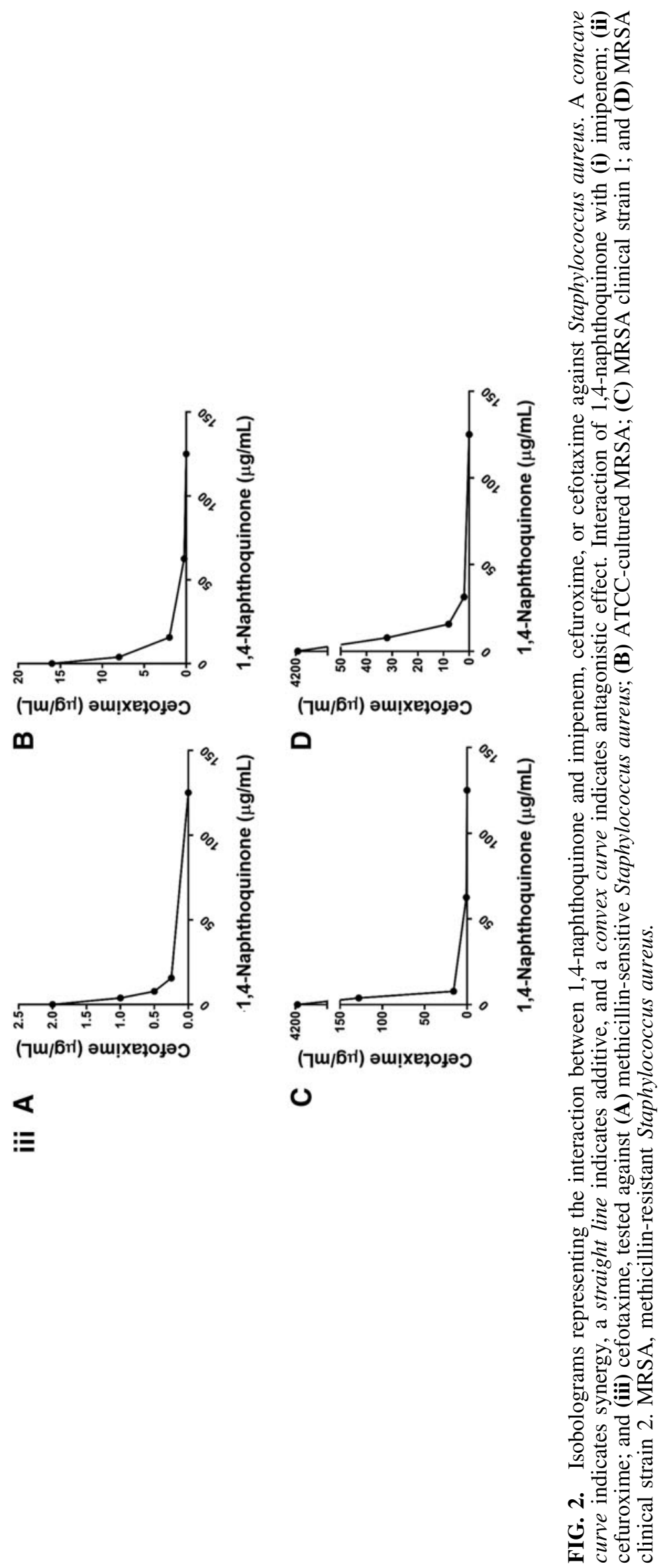


instead antagonizes the antibiotic's inhibitory activity. Interestingly, however, 1,4-naphthoquinone worked remarkably well against clinical strains of MRSA with all three combinations producing synergistic effects. The results of this study suggest that, in addition to its own broad-range antibacterial properties, 1,4-naphthoquinone could restore the susceptibility of MRSA toward certain $\beta$-lactam antibiotics. 1,4-naphthoquinone-antibiotic combinations are particularly effective against clinical isolates of MRSA, which are far stronger than ATCC-cultured ones, as they are more commonly exposed to environment challenges, including antibiotics, which promote positive selection for resistance among bacteria. Nevertheless, the pathways in the antibacterial activity of 1,4-naphthoquinone could be a novel target in $S$. aureus, as the structure of 1,4-naphthoquinone differs from that of antibiotics. The molecular basis of the synergistic, additive, or antagonistic effects of 1,4-naphthoquinone requires further studies.

\section{Conclusion}

Overall, this study demonstrated that 1,4-naphthoquinone is not only a potent antibacterial compound, but could also potentially be developed into an adjuvant to restore antibiotic function against drug-resistant bacteria, particularly MRSA. From the results, a combination therapy consisting of 1,4-naphthoquinone and cefotaxime is perhaps the most promising measure against MRSA infections as their synergy and effectiveness were proven against MSSA and all strains of MRSA tested. However, it is recommended that animal studies be conducted to assess the safety and efficacy of these combinations in in vivo settings.

\section{Disclosure Statement}

No competing financial interests exist.

\section{Funding Information}

This work was supported by International Medical University BioMedical Science Research Grant [grant numbers BMSc I-2018 (01)].

\section{References}

1. Holding, C. 2004. How bacteria fight antibiotics. Genome Biol. 5:spotlight-20040513-01.

2. Wright, G.D. 2014. Something old, something new: revisiting natural products in antibiotic drug discovery. Can. J. Microbiol. 60:147-154.

3. González-Bello, C. 2017. Antibiotic adjuvants—a strategy to unlock bacterial resistance to antibiotics. Bioorg. Med. Chem. Lett. 27:4221-4228.

4. Todd, P.A., and P. Benfield. 1990. Amoxicillin/clavulanic acid: an update of its antibacterial activity, pharmacokinetic properties and therapeutic use. Drugs 39:264-307.

5. White, A.R., C. Kaye, J. Poupard, R. Pypstra, G. Woodnutt, and B. Wynne. 2004. Augmentin(R) (amoxicillin/ clavulanate) in the treatment of community-acquired respiratory tract infection: a review of the continuing development of an innovative antimicrobial agent. J. Antimicrob. Chemother. 53:i3-20.

6. Widhalm, J.R., and D. Rhodes. 2016. Biosynthesis and molecular actions of specialized 1,4-naphthoquinone natural products produced by horticultural plants. Hortic. Res. 3:16046.

7. Klotz, L.O., X. Hou, and C. Jacob. 2014. 1,4-Naphthoquinones: from oxidative damage to cellular and inter-cellular signaling. Molecules 19:14902-14918.

8. Jin, Y.R., C.K. Ryu, C.K. Moon, M.R. Cho, and Y.P. Yun. 2004. Inhibitory effects of J78, a newly synthesized 1,4naphthoquinone derivative, on experimental thrombosis and platelet aggregation. Pharmacology 70:195-200.

9. Janeczko, M., O.M. Demchuk, D. Strzelecka, K. Kubiński, and M. Masłyk. 2016. New family of antimicrobial agents derived from 1,4-naphthoquinone. Eur. J. Med. Chem. 124: 1019-1025.

10. Ravichandiran, P., M. Masłyk, S. Sheet, M. Janeczko, D. Premnath, A.R. Kim, B.H. Park, M.K. Han, and D.J. Yoo. 2019. Synthesis and antimicrobial evaluation of 1,4naphthoquinone derivatives as potential antibacterial agents. ChemistryOpen 8:589-600.

11. Ravichandiran, P., S. Sheet, D. Premnath, A.R. Kim, and D.J. Yoo. 2019. 1,4-Naphthoquinone analogues: potent antibacterial agents and mode of action evaluation. Molecules 24:pii: E1437.

12. Sánchez-Calvo, J.M., G.R. Barbero, G. Guerrero-Vásquez, A.G. Durán, M. Macías, M.A. Rodríguez-Iglesias, J.M.G. Molinillo, and F.A. Macías. 2016. Synthesis, antibacterial and antifungal activities of naphthoquinone derivatives: a structure-activity relationship study. Med. Chem. Res. 24: 930 .

13. Ambrogi, V., D. Artini, I. de Carneri, S. Castellino, E. Dradi, W. Logemann, G. Meinardi, M. Di Somma, G. Tosolini, and E. Vecchi. 1970. Studies on the antibacterial and antifungal properties of 1, 4-naphthoquinones. Br. J. Pharmacol. 40:871-880.

14. Aly, M.R.E., M.M. Ibrahim, A.M. Okael, and Y.A.M.H. Gherbawy. 2014. Synthesis, insecticidal, and fungicidal screening of some new synthetic quinoline derivatives. Bioorg. Khim. 40:234-247.

15. Kobayashi, K., S. Nishiumi, M. Nishida, M. Hirai, T. Azuma, H. Yoshida, Y. Mizushina, and M. Yoshida. 2010. Effects of quinone derivatives, such as 1,4-naphthoquinone, on DNA polymerase inhibition and anti-inflammatory action. Med. Chem. 7:37-44.

16. Aminin, D., and S. Polonik. 2020. 1,4-Naphthoquinones: some biological properties and application. Chem. Pharm. Bull. 68:46-57.

17. Ravichandiran, P., S.A. Subramaniyan, S.Y. Kim, J.S. Kim, B.H. Park, K.S. Shim, and D.J. Yoo. 2019. Synthesis and anticancer evaluation of 1,4-naphthoquinone derivatives containing a phenylaminosulfanyl moiety. ChemMedChem 14:532-544.

18. Clinical and Laboratory Standards Institute. 2013. Performance Standards for Antimicrobial Susceptibility Testing. Wayne, PA: Clinical and Laboratory Standards Institute. ISBN: 1562387855.

19. Fratini, F., S. Mancini, B. Turchi, E. Friscia, L. Pistelli, G. Giusti, and D. Cerri. 2017. A novel interpretation of the fractional inhibitory concentration index: the case Origanum vulgare L. and Leptospermum scoparium J. R. et G. Forst essential oils against Staphylococcus aureus strains. Microbiol. Res. 195:11-17. 
20. Nair, S.V., G. Baranwal, M. Chatterjee, A. Sachu, A.K. Vasudevan, C. Bose, A. Banerji, and R. Biswas. 2016. Antimicrobial activity of plumbagin, a naturally occurring naphthoquinone from Plumbago rosea, against Staphylococcus aureus and Candida albicans. Int. J. Med. Microbiol. 306:237-248.

21. Guignard, B., J.M. Entenza, and P. Moreillon. 2005. $\beta$-Lactams against methicillin-resistant Staphylococcus aureus. Curr. Opin. Pharmacol. 5:479-489.

22. Jensen, S.O., and B.R. Lyon. 2009. Genetics of antimicrobial resistance in Staphylococcus aureus. Future Microbiol. 4:565-582.

23. Peacock, S.J., and G.K. Paterson. 2015. Mechanisms of methicillin resistance in Staphylococcus aureus. Annu. Rev. Biochem. 84:577-601.

24. Chan, E.W.L., Z.Y. Yee, I. Raja, and J.K.Y. Yap. 2017. Synergistic effect of non-steroidal anti-inflammatory drugs (NSAIDs) on antibacterial activity of cefuroxime and chloramphenicol against methicillin-resistant Sta- phylococcus aureus. J. Glob. Antimicrob. Resist. 10: 70-74.

25. Altaf, M., M. Ijaz, A. Ghaffar, A. Rehman, and M. Avais. 2019. Antibiotic susceptibility profile and synergistic effect of non-steroidal anti-inflammatory drugs on antibacterial activity of resistant antibiotics (oxytetracycline and gentamicin) against methicillin resistant Staphylococcus aureus (MRSA). Microb. Pathog. 137:103755.

Address correspondence to:

Elaine Wan Ling Chan, PhD

Institute for Research, Development and Innovation International Medical University 126, Jln Jalil Perkasa 19 Bukit Jalil, 57000 Kuala Lumpur Malaysia

E-mail: elainechan@imu.edu.my 